Memorias del VII Encuentro Nacional de Experiencias en la Enseñanza de la Biología y la Educación Ambiental y II Congreso Nacional de Investigación en la Enseñanza de la Biología

\title{
ENSEÑANZA DEL CONCEPTO DIVERSIDAD VEGETAL A PARTIR DE LA IMPLEMENTACIÓN DE UN HERBARIO VIRTUAL EN UNA INSTITUCIÓN EDUCATIVA OFICIAL DE LA CIUDAD DE NEIVA - HUILA
}

\section{TEACHING OF THE PLANT DIVERSITY CONCEPT FROM THE IMPLEMENTATION OF A VIRTUAL HERBAL IN AN EDUCATIONAL INSTITUTION OF NEIVA, HUILA}

\author{
JeisonHerley Rosero Toro ${ }^{1}$ \\ Lorena Lavao Laverde ${ }^{2}$ \\ Elías Francisco Amórtegui Cedeño ${ }^{3}$ \\ LeylaMarlenyRincón ${ }^{4}$
}

\section{Resumen}

A continuación presentamos los resultados del desarrollo de práctica pedagógica I al interior del Programa de Licenciatura en Educación Básica con Énfasis en Ciencias Naturales y Educación Ambiental de la Universidad Surcolombiana (Neiva-Colombia) llevada a cabo en una institución educativa oficial de la ciudad de Neiva.El proyecto se ejecutó en un periodo de 8 meses, con un grupo de trabajo conformado por veintiún estudiantes de sexto y cuatro de décimo grado cuyas edades oscilan entre 11 y 16 años y de estrato socio-económico 1. Para el caso de esta ponencia nos referimos particularmente a la aproximación acerca del concepto de diversidad que tienen los estudiantes y cómo estos son reflejados en su vida cotidiana. La metodología está enmarcada en una perspectiva participativa, empleando actividades de campo, laboratorio y uso de las Tecnología de la Información y la Comunicación (TIC'S). Los resultados obtenidos se enmarcan en un herbario virtual, el cual cuenta con 33 especies colectadas; ésta herramienta tecnológica fue diseñada como apoyo docente y de consulta por los estudiantes para explicar la diversidad de especies y el reconocimiento de la flora que cuenta la institución a partir de un enfoque

\footnotetext{
${ }^{1}$ Estudiante de noveno semestre del Programa de Licenciatura en Educación Básica con Énfasis en Ciencias Naturales y Educación Ambiental. Universidad Surcolombiana. U2009180406@usco.edu.co

${ }^{2}$ Estudiante de noveno semestre del Programa de Licenciatura en Educación Básica con Énfasis en Ciencias Naturales y Educación Ambiental. Universidad Surcolombiana. u2009181138@usco.edu.co

${ }^{3}$ Docente de Planta de Tiempo Completo. Programa de Licenciatura en Educación Básica con Énfasis en Ciencias Naturales y Educación Ambiental. Universidad Surcolombiana. elias.amortegui@usco.edu.co

4 Docente catedrática. Programa de Licenciatura en Educación Básica con Énfasis en Ciencias Naturales y Educación Ambiental. Universidad Surcolombiana. leylamarleny@gmail.com
} 
Memorias del VII Encuentro Nacional de Experiencias en la Enseñanza de la Biología y la Educación Ambiental y II Congreso Nacional de Investigación en la Enseñanza de la Biología

científico e investigativo. Finalmente, mostramos una forma diferente de llevar al aula el concepto de diversidad y la importancia de conocer nuestro entorno vinculando la labor investigativa en el aprendizaje del educando.

Palabras Clave: Diversidad, herbario virtual, enseñanza, Ciencias Naturales y Educación Ambiental.

\section{Abstract}

Here are the results of the development of teaching practice I into the Bachelor of Education Program with Emphasis on Basic Science and Environmental Education at the University Surcolombiana (Neiva-Colombia) held in an Educational Institution. The project was implemented over a period of eight months, with a working group made up of students of sixth and tenth grade students aged between 11 and 16 years. In the case of this paper we refer particularly to the approach on the concept of diversity with students and how these are reflected in their daily lives. The methodology is framed in a participatory approach, using field activities, laboratory and use of information and communication technologies (ICT's). The results are part of a virtual herbarium which has 33 species collected, this technological tool is designed to support teaching and consultation by students to explain the diversity of species and flora recognition to the institution from a scientific approach and research. Finally, we show a different way of bringing to the classroom the concept of diversity and the importance of knowing our environment linking research work in student learning.

Keywords: Diversity, virtual herbarium, education, Science and Environmental Education

\section{Introducción}

El Programa de Licenciatura en Educación Básica con Énfasis en Ciencias Naturales y Educación Ambiental, es un programa de formación inicial de docentes adscrito a la Facultad de Educación de la Universidad Surcolombiana (Neiva-Huila); éste, consta de nueve semestres y cuatro ámbitos de formación (Biología, Química, Física y Didáctica) que componen el núcleo común dentro del componente básico específico en el plan de estudios. Al interior del programa, se realizan dos prácticas pedagógicas llevadas a cabo al interior de octavo y noveno semestre respectivamente, realizándose la Práctica Pedagógica I en el nivel de la básica secundaria ${ }^{5}$ en una institución educativa de cualquier municipio del Departamento del Huila, en el área de Ciencias Naturales y Educación Ambiental;

5 De acuerdo a la normatividad vigente del Ministerio de Educación Nacional. 
Memorias del VII Encuentro Nacional de Experiencias en la Enseñanza de la Biología y la Educación Ambiental y II Congreso Nacional de Investigación en la Enseñanza de la Biología

mientras que la Práctica Pedagógica II se lleva a cabo en la Educación Media, particularmente en el área de Química o Física.

Para el caso de Colombia, las directrices planteadas por el Ministerio de Educación Nacional, contemplan dentro de los Estándares y Competencias para el grado sexto, el desarrollo de contenidos en torno a las diferentes concepciones científicas acerca delos seres vivos y su medio ambiente, teniendo que abarcar el concepto de diversidad como uno de los contenidostemáticos.

En éste sentido, el concepto de diversidad es fundamental para explicar, aprender y enseñar cómo funcionan las comunidades y las poblaciones dentro de un ecosistema, además de la importancia que tienen estas para el desarrollo de los seres vivos.

Para Chickering (1993) "aprender no es sentarse en clase, escuchar al profesor y memorizar aquellos conceptos asociados a la asignatura para posteriormente repetirlos. Los estudiantes deben ser capaces de hablar y escribir acerca de lo que ellos están aprendiendo y relacionarlo con otras experiencias" con lo cual se busca dejar a un lado las metodologías tradicionales y fomentar aprendizajes significativos, en donde el estudiante interrelacione sus experiencias construyendo saberes más sólidos y eficaces

Específicamente en el campo de la enseñanza-aprendizaje de la Biología, las concepciones son fundamentales debido a que los estudiantes tienen explicaciones sobre los fenómenos biológicos que difieren de los aceptados desde el punto de vista científico, formadas de formas espontánea como consecuencia de sus percepciones sobre los fenómenos naturales (Banet, 2000).

\section{Jimdo}

Es un editor web gratuito que permite la creación de páginas Web. Fundada en 2007 por los jóvenes empresarios Christian Springub, FridtjofDetzner, y MatthiasHenze en el norte de Alemania, Jimdo ha crecido hasta conseguir más de 7 millones de páginas web creadas por comunidades de usuarios en 11 idiomas y cuenta con oficinas en Hamburgo, San Francisco, Tokyo, y Shanghai, cuyo equipo de trabajo está formado por más de 15 nacionalidades.Jimdo ofrece cierta libertad ya que no se necesita manejar un lenguaje HTML para crear la plataforma. (Jimdo.com)

\section{Herbarios en la Educación}

Según Moreno (2007) la actividad de los herbarios en lo que respecta a la educación, tiene diversos y profundos anclajes, como son: proporcionar datos de 
Memorias del VII Encuentro Nacional de Experiencias en la Enseñanza de la Biología y la Educación Ambiental y II Congreso Nacional de Investigación en la Enseñanza de la Biología

localidades para organizar y planificar trabajos de campo; sirven para verificar nombres científicos; son fuente de información sobre nombres comunes y usos locales de plantas; proveen de material para ilustraciones de plantas y son centro de apoyo para la enseñanza de la botánica.

Los Herbarios virtuales nacieron como una herramienta didáctica y a disposición de toda la comunidad interesada en conocer las distintas plantas de una región, su distribución, nomenclatura, taxonomía, al igual de que también está dirigida a estudios científicos, organismos públicos, grupos ecologistas, asociaciones vinculadas con la naturaleza, o simplemente a quienes les tengan ciertos gustos por la identificación de plantas por medio de la fotografía. (Tomado de http://www.elhogarnatural.com/reportajes/Herbario.htm).

\section{Metodología}

El estudio se llevó a cabo a partir de la necesidad de buscar alternativas de enseñanza - aprendizaje del concepto de diversidad vegetal en estudiantes de sexto y décimo grado; se usaron dos líneas de trabajo, ambas enfocadas hacia las competencias específicas (interpretar, proponer y argumentar); las cuales se clasifican en: teórica y práctica; dentro del marco teórico se trataron las temáticas referentes y relacionadas a la morfología de las plantas, hábitos de vida de las mismas, características generales e inducción a la clasificación taxonómica, con el fin de establecer en los estudiantes los conocimientos básicos de diversidad con el fin de que conocieran las herramientas para el diseño del herbario virtual, aclarando que en este proceso se tuvieron en cuenta los preconceptos de los estudiantes de tal modo que se pudiera ampliar su conocimiento frente a este temática; empleando como estrategia metodológica los siguientes procesos: fase preliminar, fasede campo, fase de laboratorio, fase de aplicación y socialización de la plataforma.

- FASE PRELIMINAR:Se utilizaron encuestas con preguntas abiertas y de selección múltiple para determinar el grado de conocimiento por parte de la comunidad educativa sobre diversidad vegetal, además se aplicó una encuesta a 25 estudiantes entre los 11 y 16 años que cursan los grados sexto y décimo de la institución educativa oficial, con los cuales se trabajó durante el desarrollo del proyecto. Con la ayuda de los estudiantes se hizo el reconocimiento y la clasificación de los espacios verdes con los que cuenta la Institución Educativa. En conjunto con los estudiantes, se construyeron algunas reflexiones sobre la importanciay conservación de estas zonas verdes; y se dieron las pautas para las salidas de campo, (como son lalibreta de campo, los muestreos, las parte de las plantas que se deben colectar, entre otros.), entrevistas a los estudiantes acerca de la 
Memorias del VII Encuentro Nacional de Experiencias en la Enseñanza de la Biología y la Educación Ambiental y II Congreso Nacional de Investigación en la Enseñanza de la Biología

idea que tienen de herbario, el manejo y conocimiento de las páginas web y su capacidad de acceso a internet dentro y fuera de la institución.

- FASE DE CAMPO:a través de guías didácticas se construyeron las pautas de cómo hacer una colecta de ejemplares, partiendo de las ideas y preconceptos de los estudiantes; se dispusieron varias horas para realizar charlas, diálogos y dibujos en donde se construyeron conceptos como diversidad, especie, medio ambiente, colectas, entre otros, y cómo estos intervienen en nuestro diario vivir. Por otra parte, se realizaron presentaciones, talleres y jornadas de divulgación en donde los estudiantes eran los participantes activos. Por último, se colectó un ejemplar por especie, el secado y cosido se llevó a cabo en el laboratorio de Ciencias Naturales de la Universidad Surcolombiana con el apoyo del Herbario Surco.

- FASE DE LABORATORIO: Esta fase consistió en la visita por parte de los estudiantes al Herbario Surco, de la Universidad Surcolombiana, en donde se realizaron los procesos de secado e identificación de las colectas botánicas y la construcción de la plataforma con la información obtenida por los estudiantes.

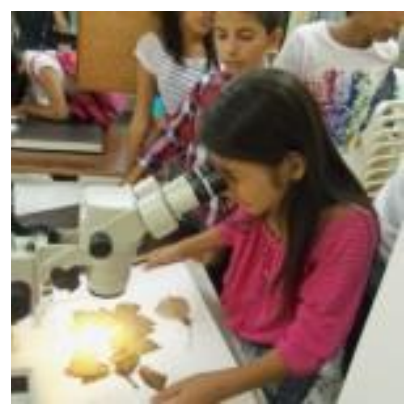

Imagen 1. Estudiante en fase de laboratorio.

- FASE DE APLICACIÓN Y SOCIALIZACIÓN DE LA PLATAFORMA: Para esta etapa se vincularon a los maestros de Ciencias Naturales de la Institución en donde se les explicó en qué consistió la plataforma y cómo ésta puede contribuir al desarrollo del tema de diversidad vegetal. Los estudiantes llevaron un proceso de aprendizaje y son ellos los que compartieron este conocimiento con sus compañeros a partir de ponencias en eventos (p.j. muestra de experiencias educativas de colegios de Neiva, organizado por la Corporación del Alto Magdalena-CAM, durante octubre de 2012), la utilización de la plataforma virtual como medio de ayuda didáctica y la participación de docentes y estudiantes en la construcción y desarrollo del herbario virtual. Por último se realizaron encuestas 
Memorias del VII Encuentro Nacional de Experiencias en la Enseñanza de la Biología y la Educación Ambiental y II Congreso Nacional de Investigación en la Enseñanza de la Biología

periódicas para contrastar las nociones construidas de diversidad vegetal a partir de las diferentes actividades.

\section{Resultados Y Discusión}

En una primera instancia indagamos qué concepto de diversidad vegetal tenían los estudiantes de grado sexto. En este sentido, hallamos que muchos de los estudiantes definendiversidad como el número de especies que compone una comunidad, mientras que conciben las comunidades más diversas, como las que hay en los bosques húmedos tropicales, presentan un mayor número de especies que se entrelazan a través de una gran variedad de interacciones ecológicas; cabe resaltar que esta definición está fuertemente relacionada con lo planteado en libros de texto para el grado tales como el Hipertexto, Santillana (2010).

Los análisis de las encuestas realizadas a los estudiantes indicaron que en los educandos hay unos vacíos del concepto de diversidad, ya que tan solo un estudiante de los 25 dio cuenta del concepto como tal, mientras que los otros solo daban cuenta del conjunto de animales, lo cual está relacionado con el hecho de que estos organismos son los que los estudiantes conciben de manera más cercana, ya que de acuerdo al contexto rural marcado en la población huilense, las familias suelen contar con mascotas, y además los estudiante viven en el barrio las Palmas, el cual presenta pocos terrenos urbanizados y en general corresponde a espacios amplios en los que puede encontrarse gran abundancia de reptiles y aves;por otra parte se pudo evidenciar que existe una falta de motivación por parte de los estudiantes en esta temática ya que consideran que es aburrida y poco interesante.

Además, se realizaron diferentes estrategias para motivar a los estudiantes a que fueran ellos los interesados en participar en el proyecto, de tal manera que a través de charlas, exposiciones y materiales tales como algunos ejemplares vegetales didácticos (muestras de plantas ya montadas con sus respectivas descripciones), fotografías y diferentes talleres fueran el impulso por aprender y construir nuevos conocimientos.

Posteriormente se llevó a cabo el reconocimiento de las zonas verdes que tenía la institución con el fin de llevar a cabo el inventario florístico; para este caso se colectaron 33 especies vegetales; durante los 8 meses de desarrollo del proyecto se llevaron a cabo trabajos ambientales como carteleras, reflexiones y discusiones que permitieran abordar un nivel de conciencia ecológica dentro y fuera de la institución, además de incorporar nuevos conceptos y la retroalimentación de la diversidad de especies con que cuenta el colegio. Luego 
Memorias del VII Encuentro Nacional de Experiencias en la Enseñanza de la Biología y la Educación Ambiental y II Congreso Nacional de Investigación en la

\section{Enseñanza de La Biología}

de lo anterior, se implementó la plataforma virtual donde se incorporó la información del proyecto.

Cabe resaltar que se tuvo participación en eventos importantes, como son el encuentro Institucional y Departamental de Semilleros de Investigación y el encuentro de Instituciones Educativas en una jornada ecológica realizada por la CAM en el centro comercial San Pedro Plaza; además se realizó una publicación en una red social en la cual RAPID COLOR GUIDES-FIELD MUSEUM integra a su plataforma nuestra página virtual, dándonos un reconocimiento a nivel internacional, en la medida que es conocida por los diferentes investigadores que hacen parte de esta herramienta tecnológica.

Para el caso de nuestro herbario virtual, fue creado bajo la plataforma de jimdo.com siendo esta una herramienta de tipo gratuita, el URL del herbario Institucional es http://herbariohumbertotafur.jimdo.com/

La plataforma del Herbario virtual contiene la información más relevante del proyecto, galerías fotográficas, talleres y participación en eventos académicos y de investigación.

La plataforma se encuentra distribuida como lo muestra la Figura 1.

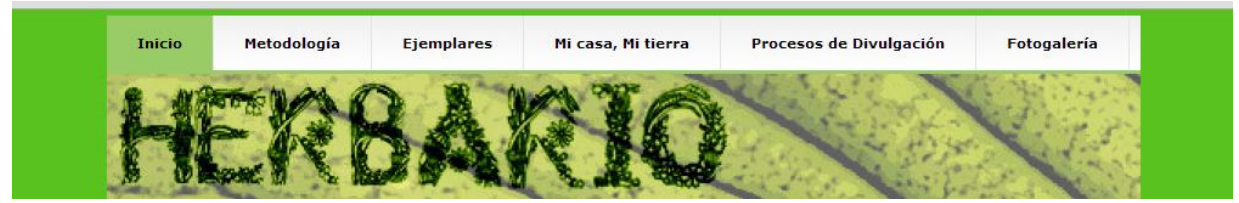

Figura 1. Plataforma virtual

En la primera pestaña de Inicio se encuentra una descripción del proyecto:

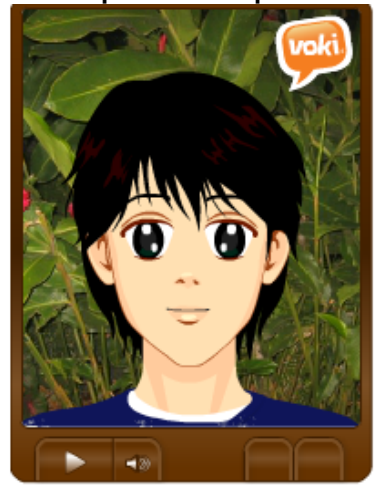

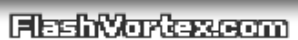

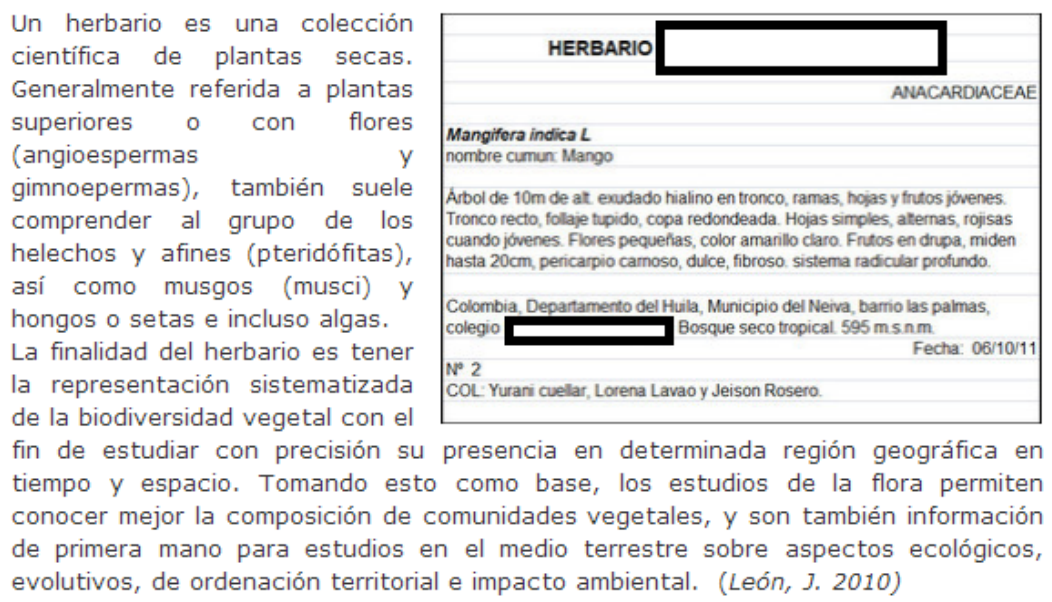

Figura 2. Pestaña Inicio del Herbario Virtual 
Edición Extra-Ordinaria. ISSN 2027-1034 P. p 486 - 495

Memorias del VII Encuentro Nacional de Experiencias en la Enseñanza de la Biología y la Educación Ambiental y II Congreso Nacional de Investigación en la

Enseñanza de la Biología

En la sección de metodología se puede consultar información más detallada de la ejecución y proceso del proyecto, como son las herramientas, las metas y los antecedentes.

\begin{tabular}{|l|}
\hline Herramientas \\
\hline Planteamiento del problema \\
\hline Evidencias \\
\hline Área de estudio \\
\hline Objetivos \\
\hline Población \\
\hline Metas \\
\hline Linea base \\
\hline Indicadores \\
\hline Antecedentes \\
\hline
\end{tabular}

\section{Metodología}

En este proyecto se usaron dos líneas de trabajo, ambas enfocadas hacia las competencias especificas, interpretar, proponer y argumentar; estas se clasifican en: teórica y práctica; dentro del marco teórico se trataron las temáticas referentes y relacionadas a la morfología de las plantas, hábitos de vida de las mismas, características generales e inducción a la clasificación taxonómica, con el fin de establecer en los estudiantes los conocimientos básicos para poner en marcha el herbario, aclarando que en este proceso se tuvieron en cuenta los preconceptos de los estudiantes de tal modo que se pudiera cambiar las concepciones que ellos tenían y ampliar su conocimiento frente al tema del Herbario; esta indagación acerca del

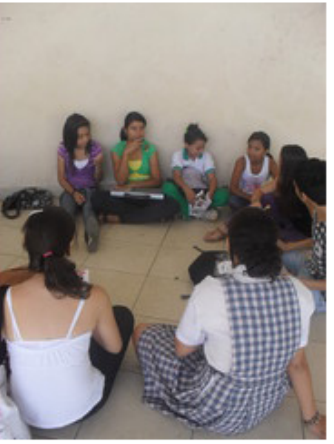
conocimiento de los estudiantes se realizo mediante una encuesta.

Figura 3. Pestaña Metodología del Herbario Virtual

En ejemplares se encuentran la colección botánica, en la primera parte hay una presentación de los ejemplares colectados; para la consulta de cada especie estos aparecen en la parte izquierda de la plataforma.

\begin{tabular}{|l|}
\hline A. purpurata \\
\hline Chlorophytum comosum \\
\hline Zea mays \\
\hline Aglaonema \\
\hline Nephrolepis exalta \\
\hline $\begin{array}{l}\text { Alocasia sanderiana. (Cara } \\
\text { de mula grande) }\end{array}$ \\
\hline $\begin{array}{l}\text { Acer palmatum. (Melena de } \\
\text { león) }\end{array}$ \\
\hline $\begin{array}{l}\text { Mirabillis expansa. (Pega } \\
\text { pega) }\end{array}$ \\
\hline $\begin{array}{l}\text { Heliconia Psittacorum. (Ave } \\
\text { del paraiso) }\end{array}$ \\
\hline
\end{tabular}

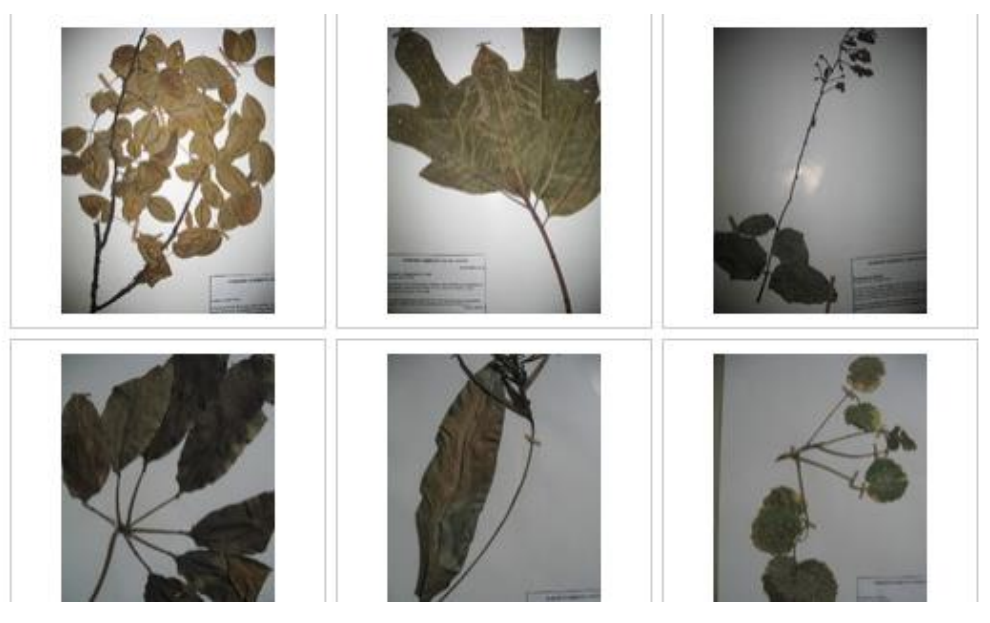

Figura 4. Pestaña Ejemplares del Herbario Virtual

En Mi casa, Mi tierra puede encontrar fotografías y dibujos como resultado de actividades llevadas a cabo en la Institución. 
Edición Extra-Ordinaria. ISSN 2027-1034 P. p 486 - 495

Memorias del VII Encuentro Nacional de Experiencias en la Enseñanza de la Biología y la Educación Ambiental y II Congreso Nacional de Investigación en la Enseñanza de la Biología

\section{Expresión artística}

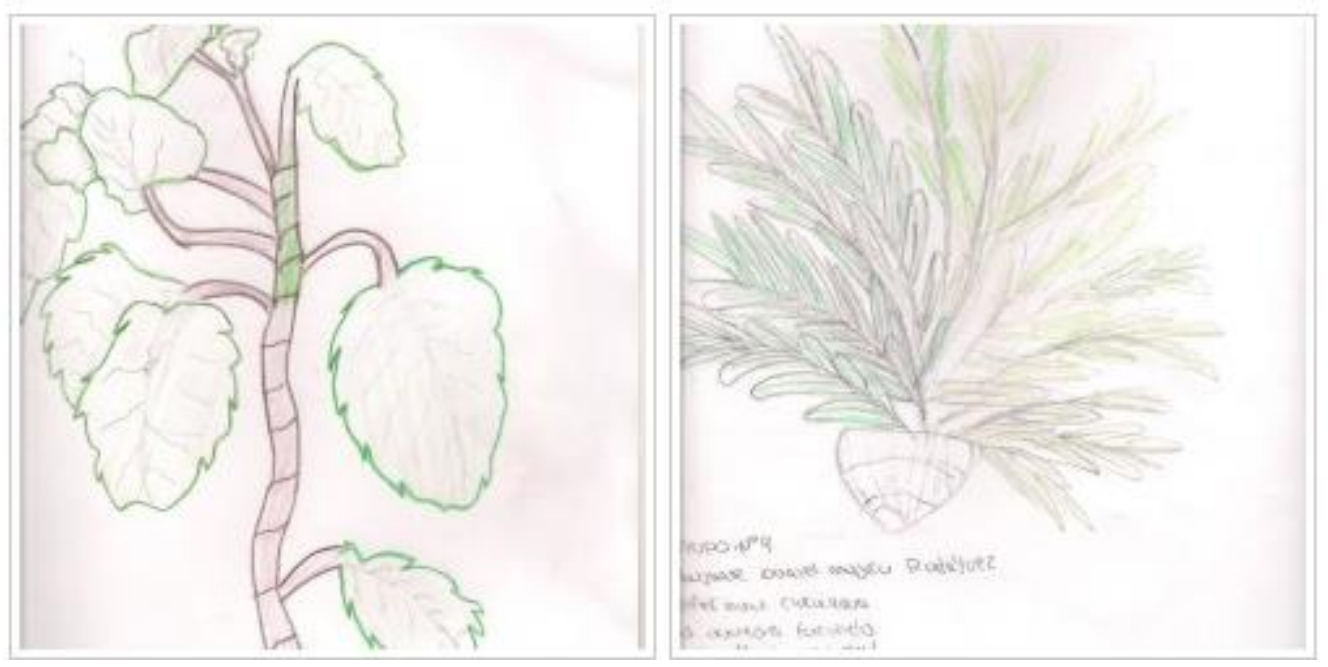

Figura 5. Pestaña Mi casa Mi tierra del Herbario Virtual

En la pestaña de Procesos de Divulgacióny Foto-galería el usuario tendrá acceso a la información fotográfica registrada en el transcurso del proyecto, evidenciando la participación en eventos y tallares.

Nuestro Encuentros

\section{Jimdo \\ Pages to the People}

Crea fácilmente una tienda online

Crea una tienda online en pocos clics. Añade los productos, establece los precios, elige las formas de pago. iListo! Prúebalo gratis en vwwitimdo.com.
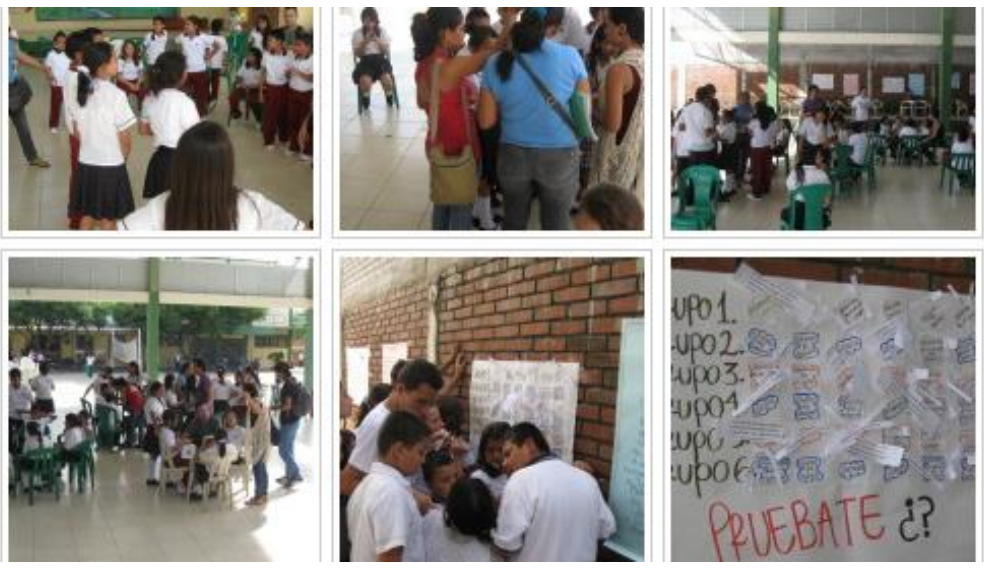

Figura 6. Pestaña Foto-galería del Herbario Virtual 
Bio-grafía Escritos sobre la Biología y su Enseñanza.

Edición Extra-Ordinaria. ISSN 2027-1034 P. p 486 - 495

Memorias del VII Encuentro Nacional de Experiencias en la Enseñanza de la Biología y la Educación Ambiental y II Congreso Nacional de Investigación en la

Enseñanza de la Biología

Es necesario comprender que las herramientas tecnológicas sirven como apoyo para el desarrollo de una clase, en esta medida el Maestros puede acceder a la plataforma y demostrar que existen formas más didácticas e investigativas para enseñar la temática de diversidad asociando los conceptos que este conlleva.

Se logra realizar el inventario florístico de la Institución Educativa y como resultado se obtiene material botánico que son ejemplos a la hora de estudiar este concepto; una plataforma virtual que se fundamenta en la investigación, en donde los estudiantes del grado decimo son los guías en la formación de los niños del grado sexto.

\section{Bibliografía}

- Carrillo, E., et al. (2010). Hipertexto Santillana. Ciencias Sexto. Editorial Santillana S. A. $174-175$.

- Chickering, A.W., Gamson, Z.F. (1993). Active Learnung: Getting Students to Work and Thinj in the Classroom. Speaking of Teaching, Stanford University Newsletter on Teaching

- Detzner, F., Henze, M \&Springub C. Plataforma Web. Jimdo.com.

- El hogar natural (2013). Licencia CreativeCommonsBy-excepto los vídeosBy-Nc-Nd. Recuperado el 21 de septiembre de 2012, de http://www.elhogarnatural.com/reportajes/Herbario.htm.

- Moreno, E. J. 2007. El Herbario como recurso para el aprendizaje de la botánica, Acta Bot. Venez. 30 (2): 13 pp. 